\title{
Philosophiques
}

\section{Libéralisme, nationalisme et pluralisme culturel}

\section{Daniel Weinstock}

Volume 19, numéro 2, automne 1992

Une nation peut-elle se donner la constitution de son choix?

URI : https://id.erudit.org/iderudit/027195ar

DOI : https://doi.org/10.7202/027195ar

Aller au sommaire du numéro

Éditeur(s)

Société de philosophie du Québec

ISSN

0316-2923 (imprimé)

1492-1391 (numérique)

Découvrir la revue

Citer ce document

Weinstock, D. (1992). Libéralisme, nationalisme et pluralisme culturel.

Philosophiques, 19(2), 117-144. https://doi.org/10.7202/027195ar d'utilisation que vous pouvez consulter en ligne.

https://apropos.erudit.org/fr/usagers/politique-dutilisation/ 


\title{
LIBÉRALISME, NATIONALISME ET
}

\author{
PLURALISME CULTUREL ${ }^{\text {I }}$
}

\author{
par \\ Daniel Weinstock
}

\begin{abstract}
A mesure qu'un peu partout à travers le monde sont en train d'émerger de nouveaux États-nations dont l'origine remonte à l'éclatement, en partie à la suite de revendications nationalistes, d'Etats multi-nationaux, un problème politique et moral qui se pose de manière urgente est celui du sort des minorités culturelles au sein de ces nouveaux Etats. Il semble inévitable que de graves conflits politiques se manifesteront entre, d'une part, les membres du groupe culturel majoritaire, dont l'un des principaux soucis continuera à être la protection politique des institutions et structures à la base de leur vie commune, et d'autre part, les membres de groupes culturels minoritaires, souhaitant continuer à vivre dans ce nouvel Etat sans avoir à abandonner leur propre culture. Les premiers veulent ériger l'État sur la base de principes nationalistes, alors que les seconds voudraient que soient mises en place des garanties politiques assurant des conditions de pluralisme culturel. Je voudrais dans ce qui suit commencer à voir dans quelle mesure ces conflits pourraient être résorbés, ou tout au moins adoucis. En gros, j'essaierai de montrer que le conflit opposant nationalistes et pluralistes culturels ne repose pas sur un désaccord de principe, mais plutôt sur la question de savoir comment partager de manière juste cette ressource perçue comme étant limitée qu'est la protection politique
\end{abstract}

1. L'auteur tient à remercier pour son appui financier le Conseil de recherches en sciences humaines du Canada pour la subvention postdoctorale qui a rendu possible cette ètude. 
de la culture. J'essaierai de révéler le terrain d'entente philosophique, souvent caché par la rhétorique politique, que partagent nationalistes et pluralistes culturels en comparant leurs positions à celle du liberalisme philosophique classique, dont nous devons l'expression contemporaine la plus importante au philosophe américain John Rawls. Je suggèrerai qu'ils sont davantage en mesure de reconnaittre le poids moral des considérations mises de l'avant par leur adversaire que ne le serait le tenant de cette branche de la tradition philosophique libérale que représente Rawls. Je tenterai finalement de montrer comment la reconnaissance de l'intérêt partagé que j'attribue aux positions nationalistes et pluralistes serait en mesure de modifier quelque peu les termes du débat qui les oppose.

Mon argument ne présuppose qu'une chose qui pourrait faire objet de litige: que l'État-nation à l'intérieur duquel ces conflits émergeront est engagé à ce que sa vie politique soit gouvernée par ce que j'appellerai des normes liberales minimales, qui, comme nous le verrons, ne requièrent pas l'adoption d'une idéologie libérale globale ou exhaustive, mais tout simplement la reconnaissance des droits politiques de base, ainsi que du principe que la justice de mesures politiques doit en grande mesure être déterminée à partir de la justice des procédures qui leur auront donné lieu. (J'aurai l'occasion, dans la troisième partie de mon exposé, de revenir là-dessus). Alors que les derniers quelques mois nous donnent des raisons de croire que cette supposition n'ira pas de soi dans le cas de certains Etats-nations nés de l'éclatement de l'empire soviétique, il semble ne pas faire problème dans le contexte québécois qui nous intéresse particulièrement aujourd'hui ${ }^{2}$.

\section{LIBÉRAUX ET COMMUNAUTARIENS:}

\section{DÉSACCORDS RÉELS OU ILLUSOIRES?}

Un des plus importants débats dans la philosophie politique contemporaine tourne autour de la question de savoir dans quelle mesure le fait que notre identité morale soit liée de

2. Pour un argument selon lequel l'adhésion des québécois aux normes libérales fondamentales ne va pas de soi, voir Stephen Schecter, « Les Quebécois et le libéralisme ", in Cité libre, 20, 1992. Pour une vision plus nuancée de l'attitude du Québec envers le libèralisme anglo-saxon, voir Charles Taylor, "Shared and Divergent Values », in R.L. Watts et D.M. Brown (eds), Options for a New Canada, Toronto, University of Toronto Press, I99. 
manière constitutive aux communautés dont nous faisons partie devrait avoir des répercussions au niveau des principes de justice que nous choisissons d'adopter pour gouverner notre vie sociale et politique. La philosophie libérale classique, dont les principaux porte-parole sont Locke, Kant, Mill et d'autres, et dont la formulation moderne la plus systématique et convaincante est l'oeuvre de John Rawls, a souvent été accusée de ne pas suffisamment prendre en considération la nature intrinsèquement sociale de l'individu, et de ne faire figurer parmi ses principes politiques de base que des droits individuels. Le fait qu'elle ait si souvent employé la fiction du contrat social afin de représenter l'origine rationnelle des principes de justice en serait la preuve: la philosophie liberale représente l'être humain comme s'il n'avait pas d'attaches communautaires constitutives, comme s'il pouvait faire entièrement abstraction, dans sa reflexion à propos de la justice sociale, de ses affiliations communautaires. Le reproche fut adressé par Hegel à Kant, et dans la philosophie contemporaine, c'est un reproche tout à fait analogue qui a èté fait à l'endroit de Rawls par des penseurs que l'on a appelés «communautariens $»^{3}$. Selon eux, l'être humain se définit en grande partie par son appartenance à une communauté, à une culture, à une manière de concevoir le plein épanouissement humain; l'État devrait donc refleter ce fait, en se faisant en quelque sorte le véhicule d'un ethos commun.

De nombreux philosophes oeuvrant dans la tradition libérale ont sans doute été coupables de ce que leur reprochent ces penseurs de la communaute. Mais il est important de voir que le constat de la nature essentiellement communautaire de l'individu n'entraine pas automatiquement la thèse politique qu'ils espèrent en dégager. En effet, nous devons distinguer deux thèses distinctes:

- la thèse psychologique du communautarisme: l'identité morale de l'individu dépend des normes, des valeurs, de la culture, de la langue, etc., de la communauté dont il est issu.

- la thèse politique du communautarisme: en vertu de la thèse psychologique, l'Etat se doit de veiller à la préservation et à

3. Voir surtout Michael J. Sandel, Liberalism and the Limits of Justice, Cambridge, Cambridge University Press, I982. Voir également Alasdair MacIntyre, After Virtue: A Study in Moral Theory, Notre Dame, University of Notre Dame Press, 1984; et Michael Walzer, Spheres of Justice: $A$ Defense of Pluralism and Equality, New York, Basic Books, 1983. 
la promotion de la structure communautaire dont dèpend l'individu pour son identité.

Une position libérale plus plausible que celle qui est d'habitude attribuée aux libéraux par les communautariens pourrait souscrire à la thèse psychologique tout en présentant des arguments qui empêcheraient que celle-ci soit perçue comme appelant automatiquement la thèse politique. Il me semble qu'un argument de ce genre se profile dans un bon nombre des écrits qui constituent la tradition philosophique libérale. Je voudrais très rapidement l'esquisser.

On reprocherait au penseur libéral de faire abstraction dans ses réflexions sur la justice de l'appartenance communautaire de l'individu. Or, l'abstraction par rapport aux traits humains jugés contingents, non de manière absolue, mais du point de vue de telle ou telle pratique humaine, est un élément essentiel du jugement humain ${ }^{4}$. Nous pouvons retenir la conviction que notre culture nous définit dans une grande mesure sans croire pour autant que cette conviction est pertinente du point de vue de la justice sociale. Nous trouvons parfaitement naturel, et nous exigeons parfois moralement, de faire abstraction de certaines facettes de notre identité dans certains types de contextes. Certaines pratiques nous imposent des critères de pertinence qui nous poussent à faire abstraction de traits qui, dans d'autres contextes, pourraient fort bien être jugés essentiels: lorsque je corrige des travaux d'étudiants, je trouve parfaitement naturel, moralement requis même, de ne faire intervenir dans mon jugement que la qualité de leurs raisonnements; lorsque des personnes se livrent à une transaction commerciale, ils feront abstraction de traits qu'ils estiment non pertinents du point de vue de cette transaction. L'omission sélective de certains prédicats dans les descriptions d'individus, étant donnés les critėres de pertinence de telle ou telle pratique, fait partie intégrante des capacités qui nous sont nécessaires lorsque nous nous livrons à la délibération pratique.

Nous pourrions ainsi reformuler la position libérale de la manière suivante: bien que la thèse psychologique du communautarisme soit vraie, elle n'est pas pertinente du point de vue de la réflexion à propos de la justice sociale.

4. Voir Onora ƠNeill, "Ethical Reasoning and Ideological Pluralism », in Ethics, 98, 1988, pp. $7 \mathrm{II}^{-} 7 \mathrm{~L} 2$. 
Cependant, le libéral aurait bien sùr à justifier son omission sélective du fait de l'appartenance culturelle des individus. Les critères pertinents selon le contexte de telle ou telle pratique ne sont pas donnés dans la nature même des choses; un argument moral est requis pour établir la pertinence ou non de tel aspect de notre identité à une pratique comme celle de la justice sociale. Encore une fois, je crois que le liberral est en mesure de répondre à cette exigence. Dans l'histoire de la pensée libérale, nous pouvons identifier deux stratégies principales cherchant à combler ce vide:

- premièrement, il y aurait une approche que j'appellerai substantielle, selon laquelle nous serions en mesure d'identifier un idéal humain d'autonomie que l'État aurait la responsabilité de promouvoir. Il aurait, en reconnaissant certains droits humains fondamentaux, à veiller à la réalisation des conditions sociales requises pour que l'individu puisse développer son autonomie. L'identification à outrance avec une communauté particulière figurerait parmi les traits que la politique aurait la tâche de surmonter.Selon cette stratégie, il ne serait pas question que l'État se donne la responsabilité de chercher à figer des formes de vie communautaires traditionnelles, celles-ci ayant justement l'effet d'empêcher le plein développement de nos capacités. Selon certaines interprétations, la philosophie politique issue de l'idéalisme allemand, de Kant à Marx, correspondrait à ce schéma ${ }^{5}$. J'essaierai de montrer dans la deuxième partie de mon essai que Rawls y souscrit également, et ce malgré les modifications qu'il a récemment apportées à sa théorie.

- selon une deuxième stratégie, qui correspondrait plutôt à la pensée de Mill et de Humboldt, c'est un souci de tolérance qui devrait nous mener à faire abstraction des affiliations communautaires particulières des individus. Cette stratégie part du constat qu'il existe dans les sociétés modernes une trop grande variété de communautés morales, culturelles, religieuses et autres pour que l'on puisse s'attendre à ce qu'une théorie de la justice axée sur une conception précise de ce que devrait être l'agent pleinement épanoui puisse s'établir sans coercition, avec le libre consentement

5. Voir Bernard Yack, The Longing for Total Revolution: Philosophical Sources of Social Discontent from Rousseau to Marx and Nietzsche, Princeton, Princeton University Press, 1986 . 
de tous les citoyens. Dans la mesure où nous voudrions tous vivre dans une société gouvernée par des principes auxquels tous pourraient donner leur accord, nous en arrivons à la conclusion que, bien que l'affiliation communautaire corresponde à un intérêt humain important, l'Etat devrait maintenir une neutralité aussi complète que possible à l'endroit des diverses conceptions du bien-être humain coexistant dans une société donnée. Nous pouvons, et nous devrions même, donner une expression sociale à notre sentiment d'appartenance à une communauté, à une culture; mais, surtout dans les conditions sociales modernes de diversité culturelle, nous devrions le faire par le biais des associations infra-étatiques de notre choix, plutôt que par le truchement de l'État et de ses institutions 6 .

Voici donc deux arguments qui se profilent dans la tradition libérale, qui pourraient tous les deux être vus comme des manières possibles de montrer pourquoi, moralement parlant, l'affiliation culturelle devrait faire partie de ces facettes de notre identité dont nous faisons abstraction lorsqu'il est question de justice sociale. Comment un philosophe communautarien seraitil en mesure d'y répondre?

Il pourrait répondre de manière fort convaincante à la première stratégie en montrant que l'autonomie individuelle dont fait l'éloge la pensée libérale n'est possible qu'étant donné un contexte social sans lequel la réalisation d'une telle capacité humaine ne serait même pas imaginable. La pensée libérale met l'accent, par exemple, sur la capacité qu'aurait l'individu de choisir les diverses composantes de ce que sera pour lui une vie riche et épanouie. Il s'agirait de montrer, à l'instar de philosophes contemporains comme Taylor, Raz et Kymlicka, que cette capacité de choix ne peut s'exercer qu'à partir des options, des opportunités et des valeurs que l'individu ne peut retrouver qu'à l'intérieur de communautés humaines concrètes. Le choix ne peut procéder sous vide; il lui faut l'oxygène que seules sont en mesure de lui fournir des formes de vie particulières. Ainsi, si l'une des responsabilitès de l'Etat est de veiller à ce qu'existent dans la socièté les

6. L'élaboration d'un libéralisme centré sur cette notion de tolérance a récemment été entreprise par Nancy L. Rosenblum, Another Liberalism: Romanticism and the Reconstruction of Liberal Thought, Cambridge, Mass., Harvard University Press, 1987, et Charles Larmore, Patterns of Moral Complexity, Cambridge, Cambridge University Press, 1987. 
conditions nécessaires pour que l'individu puisse développer son autonomie, il faut également qu'il se donne la tâche de veiller à ce que les communautés, qui en sont jusqu'à un certain point les conditions de possibilite, soient preservées ${ }^{7}$.

Un libéral ayant opté pour la deuxième stratégie évoquée plus haut serait en mesure d'accepter cet argument communautarien, mais pourrait rétorquer que le fait que le gouvernement a la responsabilité de préserver et même de promouvoir l'existence de communautés et d'associations humaines pour que l'individu puisse aller y chercher les conditions de son autonomie, n'entraîne pas du tout qu'il ait la responsabilité de protéger ces communautés particulières, celles dont le nationaliste et le pluraliste culturel réclament la protection. Il doit veiller à ce qu'il existe dans la socièté suffisamment d'associations et de regroupements pour que l'individu ait à sa disposition une gamme assez ample d'alternatives pour exercer sa capacité de choisir de manière significative. $D^{\prime}$ insister sur le fait que ce soit ces alternatives plutôt que d'autres qui lui soient disponibles violerait la condition de tolérance évoquée plus haut; pourquoi l'Etat, en veillant à la protection de formes communautaires particulières, abriterait-il certaines formes communautaires de changements qui risqueraient de survenir au terme de choix humains libres, et $\mathrm{ce}$, au détriment de celles qui risqueraient d'èmerger (et au profit de celles qui risqueraient de disparaitre) dans un « marchè » de cultures et de formes de vie libre?

Je crois que le penseur communautarien pourrait devant cette question faire appel à la distinction suivante: nous devons distinguer une conception de la neutralité politique selon laquelle, en vertu d'un souci de tolérance, l'État se doit de maintenir une neutralité envers toute conception du bien, quelle qu'elle soit, et une conception de la neutralité selon laquelle ce même souci de tolèrance impose à l'Etat la responsabilité de maintenir une attitude neutre à l'endroit des conceptions du bien sujettes à controverse. Selon cette deuxième conception,

7. Voir Charles Taylor, "Atomism ", in Philosophical Papers 2: Philosophy and the Human Sciences, Cambridge, Cambridge University Press, 1985; Joseph Raz, The Morality of Freedom, Oxford, Oxford University Press, 1986, chs. 14-15; et Will Kymlicka, Liberalism, Community and Culture, Oxford, Oxford University PTess, 1989, ch. 8.

8. C.f. Allen Buchanan, Secession: The Morality of Political Divorce from Fort Sumter to Lithuania and Quebec, Boulder, Westview Press, 1991, P.55. Voir également son « $\wedge$ ssessing the Communitarian Critique of Liberalism », in Ethics, 99, เg89, pp. $869-873$ 
l'État ne devrait pas exclure d'avance la possibilité qu'il pourrait se dégager au sein d'une societé un accord normatif tournant autour d'une idée, même très génèrale, de ce qui constitue le bien-être humain. L'État ne doit demeurer neutre que lorsque se manifeste parmi les citoyens un désaccord fondamental sur des questions de cet ordre; il doit également cependant se ménager suffisamment de souplesse pour permettre la possibilité qu'il se dégage au sein d'une société un consensus gènéralisé sur des questions normatives, et pour que la réflexion sociale sur la justice puisse se faire à partir, et non en abstraction, de ce consensus.

Nous pouvons imaginer une société dans laquelle un tel consensus se formerait autour de ce que j'ai appelé la thèse politique du communautarisme. Une societé pourrait fort bien exister à l'interieur de laquelle tous accepteraient que l'affiliation communautaire représente un intérêt individuel suffisamment important pour que l'État soit chargé de veiller à la protection des structures culturelles de ses diverses communautés. Un penseur libéral optant pour la stratégie de la tolèrance devrait pouvoir accepter que dans une telle société, le débat politique portant sur les principes de justice fondamentale devrait pouvoir se faire à partir de ce consensus fondamental sur ce qui constitue une vie pleinement épanouie. Au sein d'une telle société, les conflits politiques ne porteraient pas sur des questions d'ordre fondamental ayant trait à ce qui constitue « le bien humain »; ils auraient plutôt le caractère d'un problème de justice distributive: étant donné un nombre de communautés culturelles dont les membres acceptent tous la thèse politique du communautarisme, comment distribuer de manière juste cette ressource politique limitée qu'est la protection des structures culturelles?

Je crois que nous pourrions avec profit nous représenter de cette manière les conflits qui risqueraient d'émerger dans un Québec souverain entre nationalistes et pluralistes culturels ${ }^{9}$. Tous souhaiteraient que l'État se charge de protéger les structures culturelles qui sont à la base de leur vie commune, mais chacun imagine qu'un gain pour l'un représente nécessairement une perte pour l'autre. Comment pourrait s'entamer un dialogue sur la justice entre ces divers groupes, unis par le fait d'accepter la thèse politique du communautarisme, mais séparés par les

9. Cette manière de concevoir le conflit entre anglophones et francophones au Québec a également été suggèrée par Josée Legault, L'invention d'une minorité: les Anglo-Québecois, Montréal, Editions Boréal, 1992. 
positions incompatibles qu'ils sembleraient avoir quant à la question de la juste distribution? C'est à cette question que je me tournerai dans la troisième partie de cet essai. Je voudrais tout d'abord chercher à mettre à jour aussi clairement que possible les raisons qui me font dire qu'un tel débat doit nécessairement déborder du cadre de la pensée libérale rawlsienne.

\section{RAWLS FACE AUX REVENDICATIONS COIIECTIVES}

Dans un important livre publié en 1989, Will Kymlicka tenta de montrer que si des penseurs libéraux américains tels que Dworkin et Rawls n'avaient pas dans leurs oeuvres placé les droits culturels dans leurs listes de droits que l'Etat libéral se devait de protéger, il ne s'agissait pas là d'une manifestation de prémisses profondèment ancrées dans leurs théories, mais tout simplement d'une myopie conceptuelle causeee par leur attachement au modèle de l'Etat-nation monoculturel, ainsi que par des réflexes individualistes auxquels semblent susceptibles les philosophes libéraux. Il s'agit là cependant, selon Kymlicka, d'un oubli plutôt que d'un véritable empêchement conceptuel: les structures d'argumentation élaborées par Rawls et Dworkin afin de spécifier la liste de droits individuels que l'État libéral se doit de protéger est parfaitement en mesure de reconnaitre qu'il existe également des droits culturels dont le poids moral se situerait dans la même échelle de valeurs que les droits individuels liberraux plus traditionnels. Dans le cas de Rawls, Kymlicka se sert de l'argumentation que j'ai tout à l'heure attribuée au philosophe communautarien répondant aux objections de la position libérale substantielle: dans la mesure ou Rawls estime que les biens sociaux premiers dont il veut que l'Etat se charge de l'équitable répartition correspondent aux diverses conditions (libertés, opportunités, ressources matérielles) dont l'individu a besoin afin de développer ses principaux pouvoirs moraux, il se doit de faire figurer parmi ces biens premiers les ressources culturelles en l'absence desquelles l'idée même de ces capacités devient inintelligible ${ }^{\text {IO }}$.

La stratégie de Rawls dans $A$ Theory of Justice est la suivante il $y$ a de façon latente dans le sens commun des notions relativement stables sur ce qui constitue un agent humain pleinement épanoui. Celui-ci serait doté de deux pouvoirs moraux dont le développement constitue pour nous un intérêt dominant. Ces deux pouvoirs sont, d'une part, un sens de la justice, et d'autre 
part, la capacité de former, de réviser et de mener à bien une conception du bien, de ce qui constituera pour chacun d'entre nous une vie digne d'être vécue ${ }^{11}$. Nous souhaitons selon Rawls que les principes de la justice qui gouvernent les institutions de base de notre société soient le reflet des intérêts que nous avons à ce que soient maintenues les conditions de développement de ces capacités. Rawls imagine donc une procédure hypothétique de choix social dont la structure rend opérationnelle cet intérêt dominant. Nous voulons que nos principes de la justice soient ceux qui seraient choisis par des êtres ayant les capacités que nous estimons devoir cultiver en nous-mêmes; il s'agit donc de tenter de les dériver d'une situation de choix social où nous essayons d'approximer les processus de délibération auxquels se livreraient de tels agents. Cest le rôle que Rawls attribue à sa fameuse "position originelle $~^{12}$ : elle impose un certain nombre de contraintes quant aux informations dont disposent ces agents hypothétiques, contraintes qui ont pour rôle de refléter le sens de la justice que nous voulons incarner dans nos principes politiques. Et elle définit un certain nombre de biens sociaux premiers, conçus comme " ce qu'il est rationnel qu'un homme veuille, quelles que soient les autres choses qu'il veut $»^{13}$. Les agents hypothétiques doivent trouver des principes pour la distribution équitable de ces biens, qui reflètent en quelque sorte l'autre intérêt dominant que nous avons, celui qui consiste à nous ménager les conditions nécessaires pour pouvoir former, réviser et mener à bien un plan de vie rationnel. Quel que soit le projet que nous nous proposons d'entreprendre dans nos vies, Rawls estime que nous aurons besoin de ces biens sociaux premiers que sont droits et libertés, richesse et revenus, et respect de soi-même; en les protégeant pour chaque individu à l'aide de droits constitutionnels, nous nous assurons que l'Etat veillera à ce que chaque individu puisse développer la capacité qui le porte à avoir une conception spécifique du bien, sans que ces droits soient davantage favorables à certaines conceptions du bien plutôt que d'autres ${ }^{14}$.

II. John Rawls, Une théorie de la justice, trad. de l'anglais par Catherine Audard, Paris, Seuil, 1981, pp. 544-548; « Kantian Constructivism in Moral Theory », in The Journal of Philosophy, 77, 1980, Pp. 524-528.

12. Rawls, Op.cit., ch. 3 .

13. Ibid., p. 92.

I4. La neutralité des biens sociaux premiers de Rawls a été remise en question par Thomas Nagel, « Rawls on Justice », in Norman Daniels (ed.), Reading Rawls, Oxford, Basil Blackwell, I975, Pp. 8-9. 
Le problème dans l'argument de Kymlicka est le suivant: l'une des composantes de notre psychologie morale que nous estimons pertinente, selon Rawls, du point de vue de la recherche de principes de justice sociale est la capacité que nous avons de former, de réviser et de mener rationellement à bien une conception du bien-être humain. Dans sa Theory of Justice, nous devons attendre les dernières sections du livre avant d'apprendre comment Rawls entend développer cette notion. Selon lui, une conception du bien n'est réellement rationnellement choisie par l'individu que si rien n'exclut en principe qu'il puisse évaluer toutes les composantes de cette conception. Il écrit que:

Le projet de vie d'une personne est rationnel si, et seulement si, [...] c'est le projet qui, [,,] serait choisi sur la base d'une délibération entièrement rationnelle, c'est-à-dire avec une pleine conscience des faits pertinents et après un examen soigneux des conséquences ${ }^{15}$.

Non seulement, selon Rawls, l'agent doit-il pouvoir évaluer chaque plan potentiel à la lumière de ses désirs les plus fondamentaux, il doit aussi veiller à ce que les désirs qui le guident. dans son choix soient ceux qu'il serait encore en mesure d'affirmer étant données certaines conditions épistémiques idéales: ses désirs ne doivent pas avoir èté formés à partir de croyances factuelles erronées; ils ne doivent pas avoir été causés par des facteurs qui l'amèneraient à reconsidérer ces désirs s'il devenait pleinement conscient de ces forces causales; et ils ne doivent pas privilégier outrancièrement certains moments de sa vie au dètriment d'autres parties, peut-être plus éloignées dans le temps ${ }^{\mathrm{I}}$.

Il faut donc selon Rawls que nous maintenions à l'égard des conceptions que nous nous faisons à n'importe quel moment de notre bien une attitude hautement faillibiliste: étant donné le fait que de nouvelles informations pourraient nous parvenir qui nous amèneraient à changer d'avis quant au plan de vie qui serait le plus apte à satisfaire nos désirs les plus fondamentaux, et qui pourraient même nous mener à la reformulation de ces désirs, il s'agit d'une condition de rationalité même que nous voyions nos plans de vie comme étant toujours sujets à une condition de révisabilité ${ }^{17}$. Rawls ne croit pas que nous devrions, dans nos vies de tous les jours, réfléchir constamment à ce que serait notre

15. Rawls, Op. cit., p.449.

16. Ibid., pp. $451^{-452 .}$

17. C.f. Allen Buchanan, « Revisability and Rational Choice », in Canadian Joumal of Philosophy, 5,1975 . 
bien; la raison nous impose aussi de savoir quand les coûts associés avec le fait de prolonger notre délibération en arrivent à dépasser les bénéfices. Mais il ne s'agit pas ici des normes qui devraient nous guider dans la vie de tous les jours; ce qu'il cherche à formuler, c'est une conception de l'agent qui devrait nous guider dans la formulation de principes de justice sociale adéquats. Et c'est à ce niveau que Rawls estime que nous devrions ne pas inclure dans notre structure politique de base des principes qui feraient obstacle à ce que chaque citoyen puisse en principe se livrer à cet exercice que la rationalité délibérative lui imposerait, à savoir d'être en mesure de réviser à la lumière de nouvelles considérations les diverses composantes de sa conception du bien.

Revoyons la définition que Rawls donne des biens sociaux premiers: il s'agit de biens dont l'individu aurait besoin, quelle que soit sa conception spécifique du bien. Cette définition assure que ces biens seront neutres à l'égard des différentes conceptions de l'épanouissement humain existant dans une société à n'importe quel moment; ces réflexions nous suggèrent que l'intérêt des biens sociaux premiers pour Rawls réside également dans le fait qu'ils possèdent suffisamment de flexibilité pour pouvoir être placés au service des différentes conceptions du bien que chaque individu pourrait adopter au cours de sa vie ${ }^{\mathrm{I8}}$. Une des raisons pour lesquelles les principes que Rawls destine à la « structure de base » d'une société bien ordonnée s'occupent avant tout de répartir de manière juste les biens sociaux premiers tels qu'il les définit est que ce n'est qu'une répartition juste de ces biens qui permettra à tous de pouvoir former et mener à bien une conception du bien rationnelle, c'est-à-dire, susceptible d'être révisée.

Qu'advient-il de l'affirmation de Kymlicka selon laquelle nous pourrions sans trahir le fond de la pensée de Rawls inclure parmi les biens sociaux premiers le bien qui vient de l'association culturelle, et donc reconnaître constitutionnellement un droit à la préservation de nos structures culturelles spécifiques, qui accompagnerait les droits libéraux plus traditionnels, tels que la liberté d'expression, d'association, de culte, etc.? Je crois que mon élucidation des canons de rationalité que Rawls impose aux conceptions du bien nous permet de voir qu'il est essentiel pour lui non seulement que les individus puissent

18. Ibid., p. 406. 
choisir entre diverses conceptions du bien humain, mais que des conditions soient établies qui leur permettent, une fois leurs conceptions du bien èlaborées, de réviser ces conceptions. Or, le fait d'ériger en droit fondamental le fait que les individus puissent mettre leurs structures culturelles à l'abri des résultats qui pourraient survenir de leurs libres choix irait à l'encontre de telles conditions; il poserait des obstacles à ce que les individus soient en mesure de remettre en question l'une des composantes principales de leur conception du bien, à savoir leur appartenance à une communauté culturelle particulière. Ainsi, pour des raisons ayant des racines très profondes dans la pensée de Rawls, ayant trait à sa conception de la délibération pratique et de la formation rationnelle d'une conception du bien, il semblerait qu'il ne puisse pas, comme le prétend Kymlicka, reconnaître ce que j'ai appelé plus haut la thèse politique du communautarisme, thèse que j'ai du reste attribuée au nationalisme ainsi qu'au pluralisme culturel, à savoir que, dans la mesure où l'appartenance culturelle est cruciale dans la formation de l'identité morale de l'individu, on devrait la protéger d'un droit qui aurait dans la délibération politique un poids comparable aux droits libéraux individuels traditionnels.

Qu'en est-il pour le Rawls des dernières années, qui aurait dans ses écrits les plus récents apporté des changements fondamentaux à sa théorie afin de rendre justice, de manière plus adéquate, au fait que les individus dans les sociétés occidentales modernes se voient comme étant liés de manière constitutive à des conceptions du bien trop diverses pour que l'on puisse s'attendre à ce qu'un consensus émerge spontanément de leur sens moral commun ${ }^{19}$ ? Ces écrits ne reprèsentent-ils pas une capitulation - ou tout au moins un repli stratégique - face à la thèse politique du communautarisme ${ }^{20}$ ? Etant donnée la place importante qu'il semble maintenant reconnaitre au pluralisme moral et culturel, ne serait-il pas tout au moins voué à reconnaître une certaine validité à la position que j'ai attribuée au pluralisme culturel?

Le fait que Rawls n'entend pas modifier sa conception de ce que requiert la justice sociale se conçoit si on examine l'attitude

19. Voir surtout John luwls, « Justice as Fairness: Political not Metaphysical ", in Philosophy and Public Affairs, 14, I985; et « The Idea of an Overlapping Consensus », in Ox ford Journal of Legal Studies, 7, 1987.

20. Voir Chandran Kukathas et Philip Pettit, Rawls: $A$ Theory of Justice and its Critics, Cambridge., Polity Press, 1990, Pp. 133-142. 
qu'il adopte à l'endroit de la notion de pluralisme. Distinguons deux manières de concevoir le pluralisme: premièrement, on pourait voir le pluralisme comme la condition de réalisation d'importantes valeurs humaines. Ce n'est selon cette conception qu'en ayant un contact quotidien avec d'autres cultures, d'autres modes de vie, d'autres façons de concevoir le bien-être humain, que nous pouvons espérer éviter la sclérose de nos propres conceptions. Nous avons besoin de mettre nos propres manières de voir les choses à l'épreuve du contact avec des visions divergentes, peut-être même incommensurables, pour empêcher qu'elles se transforment en dogmes. Cette position a été avancée entre autres par John Stuart Mill, dans son célèbre On Liberty ${ }^{2 \mathrm{I}}$. Mais Mill lui-même ne tira pas les conclusions politiques qui semblaient s'imposer, à savoir qu'un des rôles de l'État devrait être de promouvoir les conditions nécessaires à ce que ce pluralisme puisse exister; il estimait que l'Etat ne pouvait en toute justice se servir de son pouvoir coercitif pour limiter la liberté d'une personne que pour l'empêcher de nuire à autrui. Dans la littérature philosophique plus récente, Joseph Raz a bien vu que le fait de concevoir le pluralisme comme une condition essentielle de la realisation d'importantes valeurs humaines doit mener à une conception plus expansive du rôle de l'Etat: selon Raz, l'Etat nuirait aux intērêts de ses citoyens en ne contribuant pas à la promotion de ces conditions. Dans la mesure où il est conçu comme ayant parmi ses responsabilités fondamentales l'autonomie de ses citoyens, l'Etat se doit de creer, ou tout au moins de promouvoir, les conditions sociales nécessaires pour que ses citoyens puissent réellement cultiver cette capacité. Le pluralisme serait selon cette optique une doctrine impliquant pour l'Etat des devoirs positifs: dans la mesure où l'on croit que le plein épanouissement humain ne peut être réalisé que lorsque les individus ont à leur portée différentes conceptions valables de ce qui constitue une vie humaine digne d'être vécue, la conclusion s'impose assez rapidement que les questions de justice sociale et les questions de sauvegarde des communautés culturelles ou d'autres types d'associations humaines ne peuvent pas être entièrement séparées ${ }^{22}$.

Distinguons cette manière de concevoir le pluralisme d'une autre qui ne fait que constater que les sociètés modernes sont marquées par une importante varièté de communautés et

21. John Stuart Mill, On Liberty, London, Dent, 1972.

22. Joseph Raz, The Morality of Freedom, chs. 14-15. 
d'associations humaines, sans accorder à ce fait une quelconque valeur politique. Il peut s'agir d'un simple fait empirique n'ayant aucune implication normative. C'est dans cette deuxième optique que John Rawls conçoit le pluralisme. Ses derniers écrits accordent, il est vrai, une place plus importante à ce qu'il appelle le « fait du pluralisme ». Il s'agit là d'une caractéristique permanente de nos sociétés modernes oủ coexiste un grand nombre de conceptions religieuses, morales, psychologiques et autres; mais ce fait ne constitue pour lui qu'une contrainte empirique à laquelle une théorie de la justice doit nécessairement se plier $^{23}$. Pour ce qui est de sa propre conception de la justice sociale, il est important de noter que Rawls ne croit pas que le fait du pluralisme devrait avoir une quelconque influence sur le contenu de sa thèrie: ses principes demeurent inchangés; le fait du pluralisme ne fait que leur imposer de plus strictes conditions de justification: il faut non seulement que leur fondement philosophique soit défendable, mais qu'ils puissent également être présentés comme compatibles avec les diverses conceptions du bien présentes dans la société.

Que le pluralisme n'ait qu'une relation externe à la theorie de la justice - qu'il s'agit d'un obstacle à surmonter plutôt que d'une valeur à intégrer - transparait dans la méthode que Rawls entend employer pour arriver à une conception de la justice sociale apte à susciter l'accord des membres d'une société pluraliste. Pour qu'une théorie de la justice satisfasse à cette condition pragmatique de justification, son élaboration doit se faire à partir de prémisses qui sont neutres par rapport aux diverses conceptions du bien. Il ne faut pas qu'elle emploie des notions dont l'enracinement dans une conception particulière les rend suspectes du point de vue des autres conceptions en présence. Pour ce faire, Rawls abandonne la méthode qui avait été employée dans $A$ Theory of Justice, laquelle tentait d'identifier, parmi les jugements moraux des individus, une certaine catégorie de jugements maximalement fiables, et il cherche plutôt à identifier au sein de la culture politique de sa société, ainsi que de ses traditions et de ses institutions, certaines idées latentes fondamentales qui pourraient servir de points de départ à l'élaboration d'une théorie, et auxquelles les membres des diverses communautés ne pourraient rien trouver à redire, dans la mesure oủ elles seraient tirées non de conceptions morales ou

23. Voir « Justice as Fairness: Political not Metaphysical », p. 225; et « The Idea of an Overlapping Consensus », pp. 4-6. 
religieuses controversées, mais d'un héritage culturel qu'ils partageraient en quelque sorte tous. Cest de cette manière que Rawls entend maintenant « dévoiler » la conception du citoyen et de ses deux pouvoirs moraux: celle-ci n'est plus vue comme latente au sens commun, mais plutôt comme intégrée à la culture politique des démocraties modernes. A partir de ces notions, la.reconstruction de la théorie peut s'opérer comme dans $A$ Theory of Justice, par le truchement de la position originelle.

Ainsi, dans ses écrits les plus récents, la thèse psychologique du communautarisme ne fait que générer certaines modifications au mode de justification des principes de justice sociale. Le fait que les individus se considèrent comme étant liés de manière constitutive à leurs communautés, le fait qu'ils pourraient même considérer que leur bien-être dépend de la préservation des structures de cette communauté, n'altère en rien la conception que nous devrions avoir du rôle de la justice. Ce sont en effet toujours les intérêts du citoyen-modèle (tel qu'il serait selon Rawls représenté dans nos traditions et notre culture politique), défini par son sens de la justice et par la capacité qu'il a de former, de réviser et de mener à bien une conception du bien, que la justice se doit de protéger ${ }^{24}$.

Pour terminer cette discussion, citons deux passages, tirés des deux différentes « phases » de son oeuvre, où il pose directement le problème des communautés susceptibles de disparaitre si elles ne disposent d'aucune protection au-delà de la simple reconnaissance des droits individuels libéraux. Selon Rawls, il sagit tout simplement d'une des conséquences non-voulues de la mise en pratique de son système que certaines communautés, ne disposant pour leur protection que des droits individuels attribués à chaque agent, n'arriveront pas à s'attirer suffisamment de membres pour que soit assuree leur survie. En I975, il écrit:

Une société bien ordonnée définit un arrière-plan équitable à l'intérieur duquel les modes de vie ont une chance raisonnable de s'établir. Si une conception du bien est incapable de se maintenir et d'acquérir des adhérents sous des conditions de liberté égale et de tolérance mutuelle, nous pouvons nous demander s'il s'agit d'une conception du bien viable, et si sa disparition doit être regrettée ${ }^{25}$.

24. Voir mon « De la prètendue incohèrence de la théorie de la justification de John Rawls », Cahiers du département de philosophie de l'université de Montréal, Printemps igg2. 
Et en I988, il écrit la chose suivante:

Ia question est la suivante: si certaines conceptions disparaissent et que d'autres ne survivent à peine dans un régime constitutionnel juste, est-ce que cela implique en soi que sa conception politique ne parvient pas à être neutre à leur endroit? [... Il semblerait qu'elle ne soit pas injuste à leur endroit, puisque les influences sociales favorisant certaines doctrines plutôt que d'autres ne peuvent être évitées. quelle que soit la conception de la justice politique. Nulle sociétè ne peut contenir toutes les formes de vie. [...] En vertu de sa culture et de ses institutions, n'importe quelle société se montrera peu propice au développement de certaines formes de vie. Mais ces nécessités sociales ne doivent pas être prises pour des parti-pris arbitraires ni pour de l'injustice ${ }^{26}$.

Ces passages indiquent bien que l'État libéral tel qu'il le conçoit n'est pas en mesure de faire quoi que ce soit pour remédier aux pressions qu'un groupe minoritaire serait susceptible de ressentir à cause des forces centripètes qu'exercerait à son endroit une culture majoritaire. Une fois les droits individuels libéraux assurés, la survie d'un groupe minoritaire ne peut dépendre que de l'attrait qu'il présente aux individus, et aux libres choix de ces individus de se joindre, ou de continuer à participer, à la vie de la communauté. Il n'y aurait aucune objection de principe, du point de vue du libéralisme rawlsien, à ce que ne survive qu'une seule conception du bien, en autant que l'hégémonie de cette conception s'est faite à l'interrieur du cadre des droits individuels traditionnels ${ }^{27}$.

\section{VERS UN LIBÉRAIISME PROCÉDURAI}

Jai tenté de démontrer dans la section précédente que le libéralisme rawlsien ètait fondamentalement hostile à la thèse politique communautarienne. Dans ses premiers écrits, cette hostilité a en grande partie été due à sa conception de la rationalité délibérative et aux notions de «biens premiers » et de « conceptions du bien » qui en découlent. Dans ses articles plus récents, sa vision du pluralisme, non comme condition de réalisation de valeurs humaines importantes, mais plutôt

25. John Rawls, “Fairness to Goodness 》, in Philosophical Review, 84, 1975. p. 549. (Traduction libre de Daniel Weinstock.)

26. John Rawls, «The Priority of Right and Ideas of the Good », in Philosophy and Public Affairs, 17, I988, pp. 265-266. (Traduction libre de Daniel Weinstock.)

27. La paradoxale incompatibilité entre pluralisme et libéralisme rawlsien a également été notée par Wesley Cragg, "Two Concepts of Community or Moral Theory and Canadian Culture », in Dialogue, 25, 1986. 
de justice, a été à la base de son hostilité à l'endroit de toute notion de droit culturel.

Le fait d'avoir établi l'incompatibilité de la version contemporaine la plus importante du libéralisme et de la thèse politique du communautarisme ne signifie pas automatiquement que libéralisme et reconnaissance de droits collectifs sont opposès de manière fondamentale. Je crois que Rawls ne se donne pas les moyens de reconnaittre l'importance que pourrait avoir la reconnaissance de droits culturels pour les intérêts fondamentaux de l'être humain parce qu'il est encore jusqu'à un certain point lié, et ce malgré ses plus récentes prises de position, à ce que j'ai appelé tout à l'heure une conception substantielle du libéralisme. Jentends par là une manière de comprendre le libéralisme considérant ses principes centraux comme l'expression de certaines croyances ayant trait à la nature de l'agent, $d u$ citoyen, de la société juste. Une conception substantielle du libéralisme (ou de n'importe quelle autre théorie politique) s'articule autour d'une thèse de forme générale: « une fois que nous aurons la conception juste de ce qu'est (l'agent, la rationalité, le citoyen) nous pourrons en déduire ce que devrait être la justice sociale ».

Je voudrais contraster cette conception substantielle avec une conception procédurale du liberralisme. Une telle conception voit le rôle du politique en général, et d'une doctrine de la justice en particulier, comme étant la résolution des conflits sociaux susceptibles de survenir à n'importe quel moment dans une société pluraliste et diversifiée, non par le biais de notions qui, implicitement ou explicitement, établissent des idéaux substantiels comme normes auxquelles doivent se mesurer tout argument et revendication politiques, mais plutôt par le moyen de normes de discussion et de délibération publiques. Une conception procédurale du libéralisme prétend qu'une solution juste à un conflit social résulterait d'un dialogue qui serait sujet à certaines contraintes, ces contraintes étant justifiées non par référence à un idéal de l'agent ou de la raison pratique auquel les individus auraient à se conformer, mais plutôt par une appréciation de ce qui est requis pour que le dialogue atteigne son but, c'est-à-dire qu'il donne lieu à un accord auquel tous pourront donner leur assentiment, et par rapport auquel tous pourront être satisfaits qu'il aura été conclu sans coercition, avec un plein égard accordé aux divers intèrêts en conflit. Ces normes émergeront non d'une conception philosophique potentiellement litigieuse de ce qu'est « la » raison pratique, mais plutôt du simple 
ront non d'une conception philosophique potentiellement litigieuse de ce qu'est « la » raison pratique, mais plutôt du simple fait de rendre explicite les conditions que doivent tous accepter les participants dans un dialogue afin que celui-ci donne lieu au type d'accord auquel tous esperrent parvenir ${ }^{28}$. Divers penseurs ont spécifié de manières différentes les normes qui doivent être imposées au dialogue pour que celui-ci donne lieu au résultat escompté. Il ne s'agit pas de dresser une liste exhaustive des contraintes que les participants devraient accepter, dans la mesure où tous se sentent engagés à règler leurs désaccords de manière à ce que tous puissent pleinement accepter le résultat. Il convient tout simplement de fournir quelques indications quant aux normes fondamentales que toute liste adequate devra inclure:

- une condition d'universabilité des raisons: tout participant devra reconnaître qu'une raison qu'il mettra de l'avant comme appuyant sa position aura le même poids moral lorsqu'avancee par un autre participant. Le poids moral des considérations à faire valoir ici dérive non du fait qu'il $\checkmark$ agit de mes considérations, mais du fait qu'il s'agit d'une considération que toute personne dans la même situation pourrait également avancer ${ }^{29}$.

- une condition de réciprocité: tout participant devrait avoir d'égales chances de faire valoir son point de vue.

- une condition de symétrie: nul participant ne doit pouvoir miser sur des considerrations qui manifestent le pouvoir qu'il détient par rapport à d'autres dans des contextes quotidiens d'interaction. Cette contrainte cherche à neutraliser

28. Cette conception procédurale s'apparente à celle de Bruce Ackerman, Social Justice in the Liberal Stale, New Haven, Yale University Press, Ig80, et "Why Dialogue? », in The Journal of Philosophy, 86, 1989; ainsi qu'a celle de Charles Larmore, Patterns of Moral Complexily, et " Political Liberalism ", in Political Theory, 18, 1990. Elle se rapproche également du projet habermasien de l'« éthique du discours». Voir l'important recueil à ce sujet édité par Seyla Benhabib et Fred Dallmayr, The Communicative Ethics Controversy, (trad. David Frisby et Shierry Weber Nicholsen), Cambridge, Mass., MIT Press, 1990.

29. Voir par exemple Thomas Nagel, The Possibility of Altruism, Princeton, Princeton University Press, 1970, ch. 10. Dans ce livre, Nagel ne lie pas cette condition d'universalisabilité à une théorie procédurale de la délibèration morale et politique; une telle théorie me semble cependant être parfaitement compatible avec ses arguments, surtout à la lumiẻre de textes plus recents tels que "Moral Conflict and Political Legitimacy », in Philosophy and Public Affairs, เ6, 1987. 
- une condition de retenue conversationnelle: tout participant doit être prèt à écarter de la conversation des considérations ayant trait à ses idéaux moraux lorsque ceux-ci ne sont pas partagés par ses interlocuteurs, et qu'aucun argument ne pourrait mener à ce qu'ils les partagent. Il est essentiel que nous reconnaissions que sur certaines questions, bien que nous soyons intimement convaincus de la justesse de nos convictions, il est raisonnable de s'attendre à ce que des personnes raisonnables et de bonne foi ne partagent pas nos points de vue. Devant une telle impasse, nous devons, afin de mener à bien le dialogue, chercher à identifier des prémisses normatives que tous puissent accepter ${ }^{31}$.

- une condition d'inclusivité bien que les participants pourraient très bien être amenés, en vertu de la condition précédente, à écarter du débat certaines positions litigieuses, il ne devrait pas y avoir d'exclusion par principe de certains types de considérations. L'agenda du dialogue ne doit être établi que par les intérêts en présence. Nous ne pouvons pas, dans une société pluraliste et hétérogène, déterminer d'avance quels intérêts devront être débattus.

- une condition de respect: les participants doivent tous se traiter dans leur dialogue comme des égaux du point de vue moral. Aucune considération ne peut être mise de l'avant qui manifesterait la conviction que certains pourraient avoir de la supériorité intrinsèque de leur position.

- une condition de respect des droits fondamentaux: aucune considération ne peut être mise de l'avant dont l'objet serait de remettre en question les droits fondamentaux de la personne, ayant trait, par exemple, à son intégrité physique, à sa sécurité, etc. On pourrait parler, à l'instar de H.L.A. Hart, d'un « contenu minimal de droit naturel ${ }^{32}$.

Une conception procédurale du libéralisme attribuera le prédicat « juste » à toute norme qui émergera d'un dialogue sujet

30. Voir par exemple Seyla Benhabib, Norm, Critique and Utopia: A Study of the Foundations of Critical Theory, New York, Columbia University Press, 1986.

31. Le rôle que joue une telle condition dans un nombre de contextes moraux et politiques a été élucidé par Stephen Holmes, « Gag Rules and the Politics of Omission », in Jon Elster and Rune Slagstad (eds). Constitutionalism and Democracy, Cambridge, Cambridge University Press, 1988.

32. Voir H.L.A. Hart, The Concepl of Law, Oxford, Oxford University Press, 196r, pp. $189^{-195 .}$ 
aux contraintes exposees ci-haut (la liste ètant non-exhaustive, pouvant inclure d'autres conditions, telles que les conditions de sincérité et de vérité sur lesquelles a insisté Habermas).

Je tiens maintenant à signaler les avantages d'une conception procédurale du libéralisme pour résoudre des conflits ayant trait aux droits culturels tels que ceux que nous voyons émerger un peu partout dans le monde à l'heure actuelle.

Premièrement, il convient de remarquer que libéralisme procédural et libéralisme substantiel intègrent de manières complètement différentes la thèse de l'individualisme. Pour un libéralisme substantiel comme celui de Rawls, l'individualisme constitue une valeur que l'État, de par ses institutions de base, se doit de protéger, parce qu'il est perçu comme étant intrinsèquement désirable. Soit en vertu des convictions morales que nous retrouvons in foro interno, (comme c'est le cas pour le Rawls première version), soit parce que ces convictions morales seraient ancrées aux fondements de notre culture politique (Rawls nouvelle version), nous aurions selon Rawls un intérêt dominant (« higher-order interest ») à protéger et à promouvoir les conditions de l'épanouissement de certaines capacités (en particulier la capacité de former, de réviser et de mener à bien une conception individuelle du bien). Cet intérêt dominant ainsi postulé détermine d'avance la liste des biens sociaux qui seront sujets à une répartition politique et à une protection constitutionnelle, les autres étant d'avance relégués à une distribution selon les lois du marché.

Le libéralisme procédural accorde lui aussi une place importante à l'individualisme. Mais il le fait non parce qu'il estime qu'une certaine manière de concevoir les capacités morales de l'individu constitue le but suprême dont la réalisation serait en quelque sorte le telos des institutions politiques, mais parce ce n'est qu'en accordant aux individus un certain nombre de droits et de libertés que pourront être satisfaites les conditions énumérées ci-haut. Pour que chacun puisse participer à part entière aux discussions politiques, il faut que chacun dispose de garanties dont le but est d'éliminer les obstacles les plus importants à ce que chaque individu puisse faire valoir son point de vue. Le libéralisme procédural intègre donc l'individualisme en tant que valeur purement politique: bien qu'il soit essentiel que l'individu dispose de certains droits individuels afin d'assurer que sa voix se fasse entendre dans les débats politiques, il est entièrement acceptable que l'individu se serve de ses droits afin 
de faire valoir un point de vue non-individualiste (dans la mesure où celui-ci ne porte pas atteinte aux autres conditions énumérées plus haut). Le libéralisme procédural ne prétend pas discerner, que ce soit dans un ciel platonicien, dans le sens commun, ou dans la tradition politique d'une sociêté, des valeurs dont la réalisation serait d'avance consacrée comme la tâche primordiale de l'État politique et de ses institutions. Il maintient que l'agenda politique d'une société, surtout d'une société où coexistent tant de marières différentes de concevoir le bien-être humain, doit être suffisamment souple pour pouvoir accomoder la diversité d'intérêts susceptibles de se manifester, sans chercher à " traduire » ces intérêts dans les termes d'une èchelle de valeurs dont la preéminence aurait été établie indépendamment d'une quelconque confirmation par le dialogue.

Revenons à la question des intėêts que des individus et des groupes de par le monde expriment en tant que membres de communautés se définissant en fonction d'un complexe de facteurs incluant langue, religion, ethnie, traditions et expérience historique partageee, et des droits correspondants qu'ils réclament pour assurer la préservation des structures à la base de ces communautés. Alors qu'un libéralisme substantiel, dans la mesure où il est construit autour de l'individualisme moral, semble incapable de reconnaître qu'il puisse y avoir des considérations morales qui auraient comme implication politique l'octroi de protections à des groupes, un libéralisme procédural, ne privilégiant pas d'emblée des modes de vie individualistes, serait parfaitement en mesure d'admettre que l'intérêt d'un individu à vivre pleinement en tant que membre d'une communauté distincte a, prima facie, autant de poids moral vis-à-vis des institutions politiques chargées de répartir les ressources sociales de manière équitable que n'auraient les intèrêts d'un individu cherchant à se ménager les conditions d'un mode de vie plus individualiste là condition, dans les deux cas, que leurs arguments ne soient pas en infraction par rapport aux contraintes dialogiques citées plus haut).

Imaginons qu'un dialogue politique respectant les normes élaborées ici se mette en marche dans un Québec indépendant entre les membres des divers groupes se réclamant tous de la thèse politique du communautarisme: tous estiment que le très fort intérêt à la survie de la communauté à laquelle il se sentent si intimement liés devrait se traduire par des mesures politiques visant à protéger les structures essentielles à la survie de cette communauté. Tous souhaitent également régler leurs conflits 
visant à protéger les structures essentielles à la survie de cette communauté. Tous souhaitent également régler leurs conflits de manière à ce que tous puissent donner leur plein assentiment à une éventuelle résolution. Ils acceptent donc les contraintes imposées à la discussion politique, dont l'objet est de neutraliser l'impact de facteurs qui, dans la vie de tous les jours, ont tendance à empêcher que la discussion donne lieu à une telle résolution.

Qu'est-ce que ces considérations nous permettent de dire sur la manière que devra procéder le débat dans un Québec souverain entre, d'une part, une majorité culturelle désirant se servir des instruments de l'État afin d'assurer la survie de sa structure culturelle, encore perçue comme étant menacée en vertu de sa position massivement minoritaire sur un continent largement anglophone où la fluidité des communications et l'interdépendance économique font fi des frontières politiques, et, d'autre part, des groupes culturels minoritaires désirant également vivre au Québec tout en maintenant intacts d'importants éléments de leurs vies communes (leurs langues, leurs religions, leurs traditions)? Le conflit entre ces groupes tient au fait que, à tort ou à raison, ils ont tendance à voir leur situation par rapport aux ressources sociales que sont les protections culturelles comme étant une sorte de « jeu à somme nulle », où les gains d'un groupe sont immédiatement perçus par les autres comme étant. de leur point de vue, des pertes. Un tel dialogue pourrait-il contribuer à la résolution, ou peut-être au moins à l'adoucissement, de ce conflit? Je voudrais suggérer deux types de réponses qui découlent assez naturellement d'une simple appréciation des normes mises de l'avant: premièrement, je voudrais voir dans quelle mesure le souci de satisfaire à la condition d'universabilité pourrait nous amener à une nouvelle appréciation des grands intérêts que partagent ces deux groupes; deuxièmement, je voudrais avancer certaines considerations qui selon moi découlent de la responsabilité particulière qui incombe au groupe majoritaire de veiller à ce que soient satisfaites ces diverses normes conversationnelles.

D'abord, notons que les partisans du multiculturalisme et ceux du nationalisme possèdent un important terrain d'entente normatif à partir duquel ils peuvent procéder: tous reconnaissent une variante de la thèse politique du communautarisme. Ils estiment tous que l'appartenance à une communauté culturelle correspond à un intérêt suffisamment important pour être l'objet de protections politiques. Ainsi, la condition d'universabilité 
des raisons, qu'ils acceptent tous par hypothèse, leur impose de reconnaitre qu'ils ne peuvent attacher une plus grande importance morale à ce que soient réalisées les conditions de satisfaction de leur intérêt qu'à ce que les membres des autres groupes puissent eux aussi trouver satisfaction de leurs intérêts communautaires. Une asymétrie morale ne semble pouvoir être établie par un groupe qu'en vertu de certains facteurs:

- premièrement, que ses membres attribuent à leur culture une valeur intrinsèque supérieure donnant une urgence morale particulière à leurs revendications; mais cela irait de toute évidence à l'encontre de la condition de respect élucidée plus haut;

- deuxièmement, de la croyance qu'un ou des groupes pourraient avoir à l'endroit d'autres groupes que ceux-ci ne constituent pas le genre d'entité à laquelle il convient d'attribuer des droits collectifs (parce qu'ils ne constituent pas une " nation », par exemple). Mais il semble arbitraire d'établir des critères très exigeants comme conditions de qualification: il me paraît suffire pour qu'un groupe puisse sattendre à ce que ses revendications « culturelles » aient un poids politique qu'il satisfasse à un certain nombre de conditions minimales, telles que la conscience qu'ont les membres du groupe de faire partie du groupe et l'importance qu'a cette affiliation pour leur identité; l'existence de structures communautaires donnant au groupe une certaine cohésion, ainsi que la volonté de ses membres de préserver ces structures de leur vie commune; le fait qu'il y ait un ou des critères relativement clairs identifiant membres de non-membres; le fait que le groupe soit d'une taille et d'une concentration suffisantes pour que sa survie soit possible sans imposer de trop grands coûts à la société ${ }^{33}$. Au Québec, diverses communautés autochtones ainsi que la communauté anglophone satisferaient à ces exigences minimales.

- troisièmement, un groupe pourrait s'attribuer un statut politique privilégié en vertu du simple principe majoritaire: puisqu'il possède le nombre de son côté, pourquoi ne pas tout simplement s'en tenir au principe démocratique voulant que la volonté de la majorité l'emporte? Du point

33. Voir Vernon Van Dyke, "Collective Entities and Moral Rights: Problems in Liberal-Democratic Thought $»$, in The Joumal of Politics, 44, 1982, pp. 32- 
de vue de la structure de dialogue mise en place ici, cet argument serait lui aussi insatisfaisant: l'idée-force du libéralisme, qu'il soit substantiel ou procédural, est qu'une communauté doit s'engager par sa constitution à mettre certaines questions, touchant aux intérêts humains centraux, à l'écart de processus majoritaires. Il reconnaît que l'opinion publique est suffisamment instable et sujette aux passions pour que certaines questions ne puissent pas moralement être réglées par elle. Dans la mesure où nous voyons la question de la protection culturelle comme une question de droits, elle doit elle aussi être placée à l'abri des processus majoritaires; s'il existe un droit à la protection de la culture, il s'agit d'un droit qui existe independamment de la volonté de la majorité. Dans le contexte d'une telle question, le fait qu'un groupe fasse appel à sa position majoritaire afin de justifier un accès privilégié aux ressources sociales nécessaires pour préserver sa culture doit être vu comme une atteinte à la condition de symétrie évoquée plus haut.

- quatrièmement, une communauté pourrait prétendre avoir un besoin plus urgent des ressources sociales en question en vertu du danger d'extinction plus immédiat auquel fait face sa communauté. Je crois que cest la seule manière de justifier une asymétrie morale parmi les divers groupes: un groupe serait justifiè à exiger un accès privilègié aux ressources en question $s^{\prime}$ il est en mesure de montrer l'urgence de la situation dans laquelle se trouve sa communauté. Il s'agirait là d'une question empirique, sans doute très difficile à régler de manière définitive, mais au moins en principe susceptible de résolution à l'aide de normes d'enquête et de démonstration que tous les groupes en présence pourraient accepter.

Jen tire la conclusion, peut-être un peu paradoxale, qu'un groupe culturel s'étant donné un Etat parce qu'il considère que l'intérêt que ressentent ses membres à ce que soit préservées ses structures communautaires se trouve moralement obligé de reconnaître que la satisfaction de ce même intérêt ne peut pas être interdite aux autres groupes culturels se trouvant sur son territoire, à moins, bien sûr, de pouvoir montrer que l'octroi de garanties politiques ayant trait à la culture pour ces groupes constituerait un danger réel pour sa propre survie en tant que groupe culturel distinct. 
J'estime que les conditions dialogiques énumérées plus haut nous permettent également de voir que des responsabilités morales particulières incombent dans une société pluraliste au groupe culturel majoritaire. Hormis le fait, signalé plus haut, qu'il doit veiller à ne pas enfreindre la condition de symétrie, il doit manifester une plus grande vigilance que les groupes minoritaires à ce que soit satisfaite la condition de réciprocité. De par sa position majoritaire, et surtout dans une situation comme ce sera la cas pour le Québec, où l'État souverain naîtra (s'il naît) de pressions nationalistes, il doit faire attention à ce que la condition de réciprocité soit satisfaite. Selon celle-ci, la justice de la conclusion d'un dialogue politique dépend en partie de ce que tous les participants concernés aient autant de chances de faire valoir leurs points de vues. Or, une culture majoritaire, s'étant donné un État souverain en partie afin d'atteindre certains objectifs de préservation culturelle, risque fort de ne pas être spontanément prédisposée à écouter les revendications culturelles de groupes minoritaires en son sein (même si la condition d'universalisation des raisons citée plus haut lui impose d'y être attentif). De plus, les membres de la culture majoritaire, puisqu'elle est davantage en mesure que d'autres de constituer des majorités législatives favorisant ses propres intérêts culturels, doit veiller à ce qu'elle ne passe pas de lois qui portent atteinte aux droits culturels des groupes culturels minoritaires, et ce même lorsque des garanties constitutionnelles protègent ces communautés d'atteintes directes à leurs droits; des lois peuvent avoir des effets indirects tout aussi dévastateurs, lorsque, par exemple, une mesure introduite avec l'intention d'apporter de nouvelles protections à la culture majoritaire a comme effet de priver une ou des cultures minoritaires de ressources dont elle a besoin pour survivie.

Il semblerait donc que dans un Québec indépendant, la majorité francophone rencontrerait paradoxalement davantage d'obstacles moraux à ce qu'elle puisse adopter des mesures favorisant la protection de sa propre structure culturelle, parce qu'elle aurait davantage de responsabilitès à veiller à ce que soient respectés les droits des minorités culturelles en son sein, à ce que ceux-ci puissent continuer à être des participants à part entière dans le débat politique de la société québécoise, et donc, à ce que les normes conversationnelles favorisant des résolutions noncoercitives de conflits soient en place. Mais je crois que cette responsabilité s'impose: le pouvoir politique entraîne des responsabilités envers ceux que le pouvoir rend vulnérables. Alors que la 
majorité québécoise a pu jusqu'à un certain point se prévaloir de son statut minoritaire au sein de cette entité politique plus vaste qu'est le Canada afin d'exiger des prérogatives spéciales lui permettant, en tant que minoritè culturelle, de se préserver des dangers que lui imposait ce statut minoritaire, la transformation du statu quo politique transforme le statu quo moral, de telle sorte qu'elle se doit de témoigner à l'endroit des groupes minoritaires résidant en son sein le même égard qu'elle aurait voulu que la majorité culturelle canadienne témoigne au sien.

Je reconnais que ces quelques remarques n'ont qu'un statut provisoire, et qu'elles n'ont nullement été prèsentées de manière systématique. Je crois néanmoins que le dèbat politique bénéficierait énormément de ce que les divers groupes en présence en arrivent à prendre conscience du fait qu'ils sont liés par le fait de partager une conception particulière de ce qui constitue le bienêtre humain. Chacun des groupes en présence devra réaliser qu'il se doit d'être attentif aux droits culturels revendiqués par les autres, puisque ceux ci ne sont que la manifestation du même intérêt fondamental qu'il cherche lui-même à protéger à travers les institutions politiques. Cette prise de conscience n'ôtera peut-être pas grande chose au fait qu'ils se retrouveront néanmoins devant un problème de distribution de cette ressource limitée que sont les protections culturelles. Mais encore là, il se pourrait qu'elle ait des effets salutaires; par opposition aux ressources tangibles, la mesure dans laquelle de telles protections constituent effectivement une ressource limitée par rapport à laquelle les groupes en présence se retrouvent dans un « jeu à somme nulle » dépend en grande mesure de la perception qu'ils en ont. La prise de conscience sur laquelle j'ai voulu insister ici suscitera peut-être de ces adversaires politiques, que risqueraient d'être dans un Québec indépendant les nationalistes et les groupes culturels minoritaires, la croyance qu'un gain politique pour les uns ne constitue pas nécessairement de facto une perte politique pour les autres, et qu'entre personnes de bonne foi il existe suffisamment de "place » pour pouvoir croire que peut coexister de manière non conflictuelle une pluralité d'intérêts culturels différents. Divers groupes unis par le fait de vouloir s'assurer les conditions requises pour la satisfaction de leurs intérêts culturels seraient peut-être davantage disposès à faire preuve d'imagination politique que ne le seraient des groupes qui auraient l'impression de faire face à un refus systématique de reconnaître la validite et le poids moral de leurs revendications culturelles. 
J'ai tenté dans cet essai de faire trois choses: premièrement, j'ai voulu montrer que malgré leurs diffèrends politiques, nationalistes et pluralistes culturels partagent une conception sous-jacente du rôle que la communauté joue dans la formation de l'identité de l'individu, et qu'en vertu de ce fait, la protection de la structure de la communauté culturelle pertinente devient pour eux un important objectif politique. Deuxièmement, j'ai voulu montrer que malgré les efforts de philosophes tels que Kymlicka, le libèralisme rawlsien n'est pas en mesure de reconnaitre les droits culturels que nationalistes et pluralistes culturels jugent essentiels. Ses premiers ecrits privilégient une conception de la rationalité délibérative et une conception du bien qui exclut de l'agenda de la justice sociale toute considération collective, reléguant celle-ci à l'opération du marché. Dans ses plus récents articles, l'hostilité de Rawls envers toute notion de droit collectif ou culturel se manifeste par l'attitude qu'il adopte à l'endroit du pluralisme, qu'il voit comme étant un obstacle à la justification de sa théorie de la justice, plutôt que comme la condition de réalisation d'importantes valeurs humaines. Troisièmement, après avoir expliqué l'hostilité d'une certaine branche de la tradition libèrale en des termes plus généraux par le fait qu'elle véhicule ce que j'ai appelé une conception substantielle du libéralisme, j'ai indiqué certaines raisons de préférer une conception procédurale. J'ai ensuite essayé de montrer qu'une telle conception du libéralisme était en mesure d'accomoder le type de droit collectif revendiqué par nationalistes et pluralistes culturels, étant admis que les normes qu'elle impose au dialogue politique impose certaines responsabilités aux groupes culturels majoritaires.

\section{Columbia University/Université de Montréal}

